\title{
Atomic hydrogen bridge fueling NGC 4418 with gas from VV $655^{\star, \star \star}$
}

\author{
E. Varenius ${ }^{1}$, F. Costagliola ${ }^{1}$, H.-R. Klöckner ${ }^{2}$, S. Aalto ${ }^{1}$, H. Spoon ${ }^{3}$, I. Martí-Vidal ${ }^{1}$, J. E. Conway ${ }^{1}$, \\ G. C. Privon ${ }^{4,5}$, and S. König ${ }^{1}$ \\ 1 Department of Space, Earth and Environment, Chalmers University of Technology, Onsala Space Observatory, \\ 43992 Onsala, Sweden \\ e-mail: varenius@chalmers.se \\ 2 Max-Planck-Institut für Radioastronomie, Auf dem Hügel 69, 53121 Bonn, Germany \\ 3 Cornell Center for Astrophysics and Planetary Science, Space Sciences Building, Ithaca, NY 14853, USA \\ ${ }^{4}$ Instituto de Astrofísica, Facultad de Física, Pontificia Universidad Católica de Chile, Casilla 306, Santiago 22, Chile \\ 5 Departamento de Astronomía, Universidad de Concepción, Concepción, Chile
}

Received 30 September 2016 / Accepted 11 September 2017

\begin{abstract}
Context. The galaxy NGC 4418 harbours a compact $(<20 \mathrm{pc})$ core with a very high bolometric luminosity $\left(\sim 10^{11} L_{\odot}\right)$. As most of the galaxy energy output comes from this small region, it is of interest to determine what fuels this intense activity. An interaction with the nearby blue irregular galaxy VV 655 has been proposed, where gas acquired by NGC 4418 could trigger intense star formation and/or black hole accretion in the centre.

Aims. We aim to constrain the interaction hypothesis by studying neutral hydrogen structures that could reveal tails and debris connecting NGC 4418 to the nearby galaxy VV 655.

Methods. We present observations at $1.4 \mathrm{GHz}$ with the Very Large Array (VLA) of the radio continuum as well as emission and absorption from atomic hydrogen. Gaussian distributions are fitted to observed HI emission and absorption spectra. We estimate the star formation rates (SFRs) of NGC 4418 and VV 655 from the $1.4 \mathrm{GHz}$ radio emission and compare them with estimates from archival $70 \mu \mathrm{m}$ Herschel observations.

Results. An atomic HI bridge is seen in emission, connecting NGC 4418 to the nearby galaxy VV 655. An HI tail is also seen extending south-west from VV 655. While NGC 4418 is bright in continuum emission and seen in HI absorption, VV 655 is barely detected in the continuum, but shows bright $\mathrm{HI}$ emission $\left(M_{\mathrm{HI}} \sim 10^{9} M_{\odot}\right)$. We estimate SFRs from the $1.4 \mathrm{GHz}$ continuum of $3.2 M_{\odot} \mathrm{yr}^{-1}$ and $0.13 M_{\odot} \mathrm{yr}^{-1}$ for NGC 4418 and VV 655, respectively. Systemic HI velocities of $2202 \pm 20 \mathrm{~km} \mathrm{~s}^{-1}$ (emission) and $2105.4 \pm 10 \mathrm{~km} \mathrm{~s}^{-1}$ (absorption) are measured for VV 655 and NGC 4418, respectively. Redshifted HI absorption is seen $\left(v_{\mathrm{c}}=2194.0 \pm 4.4 \mathrm{~km} \mathrm{~s}^{-1}\right)$ towards NGC 4418, suggesting gas infall. North-west of NGC 4418, we detect HI in emission, blueshifted $\left(v_{\mathrm{c}}=2061.9 \pm 5.1 \mathrm{~km} \mathrm{~s}^{-1}\right)$ with respect to NGC 4418, consistent with an outflow perpendicular to the galaxy disk. We derive a deprojected outflow speed of $178 \mathrm{~km} \mathrm{~s}^{-1}$, which, assuming a simple cylindrical model, gives an order-of-magnitude estimate of the HI mass outflow rate of $2.5 M_{\odot} \mathrm{yr}^{-1}$.

Conclusions. The morphology and velocity structure seen in $\mathrm{HI}$ is consistent with an interaction scenario where gas was transferred from VV 655 to NGC 4418. We argue that the galaxies have passed each other once, about $190 \mathrm{Myr}$ ago, and that this interaction has caused the tidal HI bridge and HI tail seen today. Some gas is falling towards NGC 4418, and may fuel the activity in the centre. We interpret blueshifted HI-emission north-west of NGC 4418 as a continuation of the outflow previously reported on smaller scales, powered by star formation and/or black hole accretion in the centre.
\end{abstract}

Key words. galaxies: interactions - galaxies: irregular - galaxies: starburst - quasars: individual: NGC 4418 galaxies: individual: VV 655

\section{Introduction}

The galaxy NGC 4418, at a distance of $34 \mathrm{Mpc}$, has a nucleus that is very bright in the infrared, but emits significantly less radio emission than expected from the far-infrared (FIR) radio correlation for star-forming galaxies (Yun et al. 2001). Since the study of Roche et al. (1986), multiple studies have presented observational evidence that is compatible with both a

\footnotetext{
* The movie associated to Fig. 4 is available at http://www . aanda.org

$\star \star$ The radio continuum image and the spectral cube presented in Fig. 1 are only available at the CDS via anonymous ftp to cdsarc.u-strasbg. fr (130.79.128.5) or via http://cdsarc.u-strasbg.fr/viz-bin/qcat?]/A+A/607/A43
}

radio-weak AGN and/or a young starburst (see Sakamoto et al. 2013; Costagliola et al. 2013, and references therein).

Redshifted absorption lines have been found towards the galaxy nucleus, for instance in the FIR OI and OH lines by González-Alfonso et al. (2012) with Herschel ${ }^{1}$, and in $21 \mathrm{~cm}$ by Costagliola et al. (2013) with the Multi-Element Radio Linked Interferometer Network (MERLIN), indicating infall of molecular and atomic gas. Sakamoto et al. (2013) also reported a U-shaped feature visible in the optical extending towards the north-west, that is, along the rotation axis of the disc, which they interpret as an outflow.

\footnotetext{
1 Herschel is an ESA space observatory with science instruments provided by European-led Principal Investigator consortia and with important participation from NASA.
} 
The NGC 4418 nucleus contains a Compton-thick compact core $(<20 \mathrm{pc})$ surrounded by molecular gas (Sakamoto et al. 2013). The nucleus is deeply obscured, as shown by the deep $9.7 \mu \mathrm{m}$ silicate absorption feature (Roche et al. 1986; Spoon et al. 2001) and the overall resemblance to an embedded protostar in the mid-IR (Spoon et al. 2001). If a starburst is powering the source, it is very deficient in polycyclic aromatic hydrocarbon (PAH) emission, likely caused by extreme conditions and extinction in the obscuring medium around the star-forming regions. The $\mathrm{H} \alpha$ emission from NGC 4418 is limited to the nucleus (Lehnert \& Heckman 1995, their Fig. 5), suggesting that the rest of the galaxy either lacks OB stars capable of exciting HII regions, or that HII gas is absent from the disc.

ALMA observations show a very rich spectrum of molecules in an environment similar to the nuclei of the extreme starburst galaxy Arp 220 (Costagliola et al. 2015). Costagliola et al. (2013) argued that if a central starburst were responsible for all the energy output, it must be 3-10 Myr old and have a star formation rate $>10 M_{\odot} \mathrm{yr}^{-1}$. The complex radio morphology reported by Varenius et al. (2014) indeed argues in favour of a significant starburst component. If this is the case, what triggered this recent starburst in the centre of NGC 4418?

Roche et al. (1986) noted that NGC 4418 may be interacting with the nearby galaxy VV 655 (also known as MCG+0032-013) about $3^{\prime}$ south-east of NGC 4418. VV 655 has blue colours, an irregular structure, and emission lines consistent with vigorous star formation (Colless et al. 2003; Alam et al. 2015). Although this possible companion galaxy is shown by Kawara et al. (1990), their Fig. 1, and mentioned by Evans et al. (2003) and Costagliola et al. (2013), no clear evidence has been presented for any interaction between NGC 4418 and VV 655.

In this paper we present radio observations taken with the VLA to search for neutral hydrogen structures connecting NGC 4418 to VV 655. The paper is organised as follows. In Sect. 2 we describe the observations and data reduction. In Sect. 3 we present the results of analysis and modelling performed using the data. The results are discussed in Sect. 4 and finally summarised in Sect. 5. We assume a distance to NGC 4418 (and VV 655) of $34 \mathrm{Mpc}$, that is, $1^{\prime \prime}=161 \mathrm{pc}$, as given by Sakamoto et al. (2013).

\section{Observations and data reduction}

We present data from the VLA taken in D configuration in project AS748 (PI: H. Spoon) on 2003-03-21 with 5.3 h on source. The parallel hands (RR, LL) were correlated with phasecentre RA $12^{\mathrm{h}} 26^{\mathrm{m}} 59.750$ and Dec $-00^{\circ} 53^{\prime} 31.50^{\prime \prime}$ and the data stored in the archive with time resolution of $30 \mathrm{~s}$ and spectral resolution of $48.8 \mathrm{kHz}$ per channel. The total bandwidth covered 63 channels, centred on $1.41017559 \mathrm{GHz}$.

The data were edited and calibrated in a standard manner using Astronomical Image Processing System (AIPS; Greisen 2003) version 31Dec15. The Python-based interface to AIPS, ParselTongue (Kettenis et al. 2006), was used to script the calibration. The flux scale and bandpass was calibrated using $3 \mathrm{C} 286$, which was assumed to be $15.1025 \mathrm{Jy}$ at the centre of the band as calculated by the AIPS task SETJY. The visibility phases were referenced to the calibrator J1150-0023 at RA $11^{\mathrm{h}} 50^{\mathrm{m}} 43.8707^{\mathrm{s}}-00^{\circ} 23^{\prime} 54.204^{\prime \prime}{ }^{2}$, found to be $2.94 \mathrm{Jy}$. The corrections derived from 3C 286 and J1150-0023 were applied to

\footnotetext{
2 Within 1 milliarcsecond of the current catalog position at http:// astrogeo.org/calib/search.html
}

NGC 4418. Visual inspection revealed HI emission in the channel range 16-53. The remaining channels were used to fit and remove the continuum in the UV-domain using the task UVLSF in AIPS.

The continuum-subtracted data were imaged using the task CLEAN in CASA 4.6 with Briggs weighting (robust $=1$ ) using baselines $>300 \lambda$ (to filter out far-away interfering sources). During cleaning, the data were gridded in velocity (using the optical velocity definition), and effects of the Earth's rotation and movement with respect to the solar system barycentre were corrected for assuming a HI rest-frequency of $1420.405751 \mathrm{MHz}$. A primary-beam corrected image cube was obtained of the Stokes I emission with image pixel size $4^{\prime \prime}$. The spectral cube had a velocity resolution of $10 \mathrm{~km} \mathrm{~s}^{-1}$ and RMS noise of $\sim 0.4 \mathrm{mJy}^{-1}$ beam $^{-1}$ in each velocity channel. A common synthesized restoring beam of $61.5^{\prime \prime} \times 50.6^{\prime \prime}$ with position angle (PA) $-1.19^{\circ}$ was used for all velocity channels.

We also obtained a $1.4 \mathrm{GHz}$ continuum image by deconvolving the line-free output from UVLSF using the multi-frequency synthesis gridding offered by CLEAN. The continuum image RMS noise was $0.2 \mathrm{mJy}^{-1}$ beam $^{-1}$ with a synthesized beam of $61.5^{\prime \prime} \times 51.0^{\prime \prime}$ with PA $-1.34^{\circ}$.

To obtain an independent estimate of the SFR, we checked the $70 \mu \mathrm{m}$ flux densities of NGC 4418 and VV 655 as observed by Herschel PACS (Pilbratt et al. 2010; Poglitsch et al. 2010) using data from the Herschel Science Archive. We used data taken 2011-07-17 towards NGC 4418 with the scan id 1342224340, available as a FITS image (with a calibration level of 2.5).

\section{Results}

In this section we present the results of analysing the continuum and spectral line images. We note that both images are available via the CDS.

\subsection{4 GHz continuum}

The $1.4 \mathrm{GHz}$ continuum is presented in Fig. 1a as contours overlaid on a three-colour ( $i, r, g$ band) image made from the Sloan Digital Sky Survey (SDSS) ${ }^{3}$. In addition to NGC 4418, four other sources are visible above $4 \sigma$ in Fig. 1a, which are all identified as compact $\left(<5^{\prime \prime}\right)$ sources with counterparts in the FIRST Survey (Becker et al. 1995) with the same flux density as measured in this work. Unfortunately, we did not find any redshifts for these sources, which could prove that they are background sources. However, given that they are compact, having no clear relation to either NGC 4418 or VV 655 (i.e. no extensions or alignments that could suggest e.g. AGN jet hot spots), and do not show any HI emission in Fig. 1b (i.e. indicate significantly different redshift), we assume that they are background sources.

Assuming the eastern extension is a background source, a fit of a Gaussian intensity distribution to the image finds NGC 4418 to be unresolved with an integrated flux density of $40 \mathrm{mJy}$, in good agreement with the $38.5 \mathrm{mJy}$ measured by Baan \& Klöckner (2006) using the VLA. As noted by Costagliola et al. (2013), this is also in agreement with the $38 \mathrm{mJy}$ measured with MERLIN, which indicates that there is no significant $1.4 \mathrm{GHz}$ emission on scales larger than the $0.5^{\prime \prime}$ component sampled by MERLIN (Costagliola et al. 2013, their Fig. A.1).

\footnotetext{
3 Using data from SDSS data release 12 (Alam et al. 2015) available via http://dr12.sdss3.org/mosaics/
} 


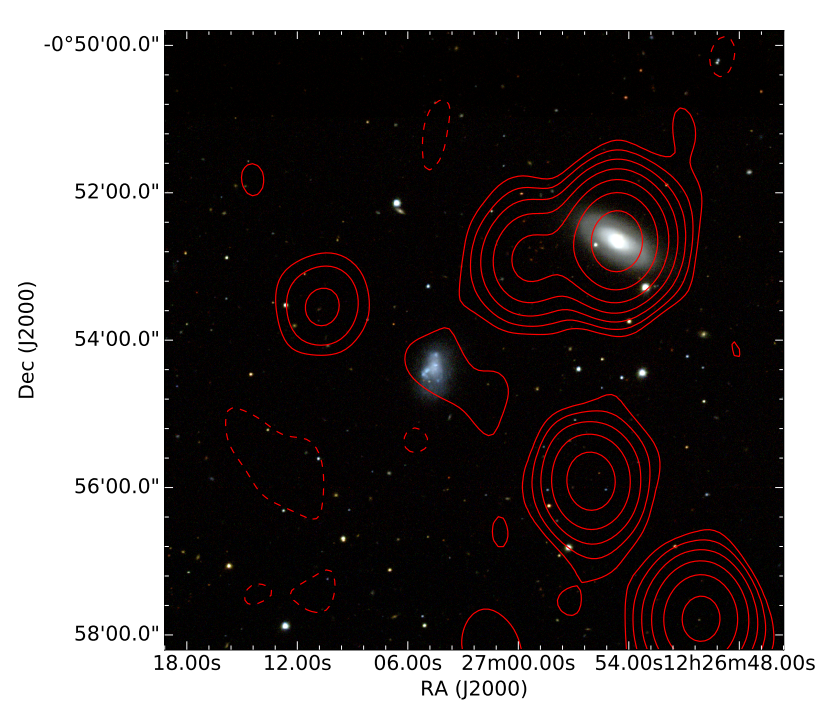

(a) $1.4 \mathrm{GHz}$ continuum

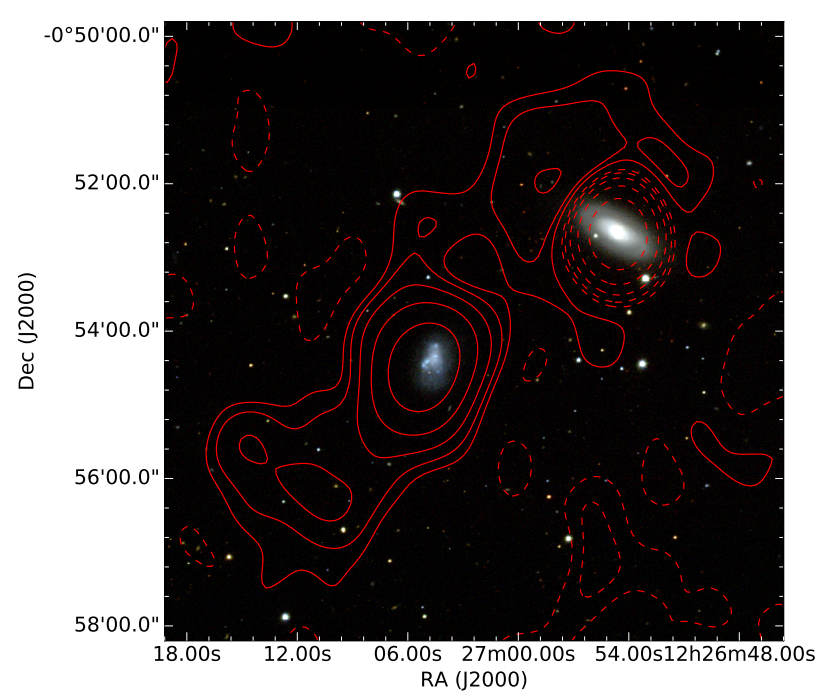

(b) HI moment 0

Fig. 1. Panel a: $1.4 \mathrm{GHz}$ continuum plotted as contours of $(-2,2,4,8,16,32,64,128) \times 0.2 \mathrm{mJy}$ beam $^{-1}$ overlaid on a three-colour SDSS image (using the $i, r, g$ bands). NGC 4418 is the white disc in the top-right part of the image, and VV 655 is the blue irregular galaxy near the image centre. In addition to NGC 4418, four other sources are visible above $4 \sigma$, as noted in Sect. 3.1. VV 655 is barely detected at $3 \sigma$ in the radio continuum. Panel $b$ : moment- 0 map of the continuum-subtracted HI (in emission and absorption) with contours at $(-32,-16,-8,-4,-2,2,4,8,16,32) \times 35 \mathrm{mJy}_{\text {beam }}^{-1} \mathrm{~km} \mathrm{~s}^{-1}$.

\subsection{Star formation rate estimates}

The galaxy VV 655 is barely detected at the $3 \sigma$ level in our $1.4 \mathrm{GHz}$ continuum image (Fig. 1a), that is, $L_{1.4 \mathrm{GHz}} \sim 8.2 \times$ $10^{19} \mathrm{~W} \mathrm{~Hz}^{-1}$. When we use Eq. (6) by Bell (2003), this corresponds to a star formation rate (SFR) of $0.13 M_{\odot} \mathrm{yr}^{-1}$, although Bell (2003) states uncertainties as large as a factor of 5 for galaxies of this low luminosity. For comparison, using the same equation given the $1.4 \mathrm{GHz}$ flux density of $40 \mathrm{mJy}$ $\left(5.53 \times 10^{21} \mathrm{~W} \mathrm{~Hz}^{-1}\right)$, we obtain an integrated radio SFR of $3.2 M_{\odot} \mathrm{yr}^{-1}$ for NGC 4418 .

From the Herschel 70 um image we measure $125 \mathrm{mJy}$ associated with VV 655, which assuming the same distance to VV 655 as for NGC 4418 of $34 \mathrm{Mpc}$, translates into $L(70 \mu \mathrm{m})=7.39 \times$ $10^{41} \mathrm{erg} \mathrm{s}^{-1}$. Calzetti et al. (2010) presented a relation to estimate the SFR from $70 \mu \mathrm{m}$ emission. While they did not claim accurate SFR estimates for individual galaxies, we used their Eq. (22) to obtain an order-of-magnitude estimate for VV 655, which is $\operatorname{SFR}(70 \mu \mathrm{m}) \sim 0.0435 M_{\odot} \mathrm{yr}^{-1}$. This is within a factor of 3 from the $1.4 \mathrm{GHz}$ estimate. We note, however, that VV 655 is below the validity limit of $1.4 \times 10^{42} \mathrm{erg} \mathrm{s}^{-1}$ stated by Calzetti et al. (2010) and therefore this value should be interpreted with caution. For comparison, using the same equation with the $39.3 \mathrm{Jy}$ $\left(2.33 \times 10^{44} \mathrm{erg} \mathrm{s}^{-1}\right)$ measured at $70 \mu \mathrm{m}$ for NGC 4418, we estimate $\operatorname{SFR}(70 \mu \mathrm{m}) \sim 13.7 M_{\odot} \mathrm{yr}^{-1}$. NGC 4418 is known to be radio weak relative to the FIR-radio correlation (Yun et al. 2001), so an $\operatorname{SFR}(70 \mu \mathrm{m})>\operatorname{SFR}(1.4 \mathrm{GHz})$ is expected.

\subsection{HI in emission and absorption}

Figure $1 \mathrm{~b}$ shows the HI emission and absorption as a moment0 map, produced by the task immoments in CASA. NGC 4418 is surrounded by $\mathrm{HI}$ seen in emission outside a distance of about $1^{\prime}$ from the centre, and seen in absorption inside this radius (see Fig. 1b). HI emission is prominent towards VV 655 and in regions between the two galaxies. The moment- 0 peak of absorption is $-52 \mathrm{mJy}_{\text {beam }}^{-1} \mathrm{~km} \mathrm{~s}^{-1}$, towards the centre of NGC 4418 and the peak of emission is $2.0 \mathrm{Jy}^{\text {beam }}{ }^{-1} \mathrm{~km} \mathrm{~s}^{-1}$, towards the centre of VV 655. A bridge of emission seems to connect VV 655 and NGC 4418, although this projection does not prove a physical connection - see further Sect. 3.3.2. Finally, there is prominent emission south-east of VV 655 with a peak of $0.34 \mathrm{Jy}_{\text {beam }}^{-1} \mathrm{~km} \mathrm{~s}^{-1}$.

The velocity structure of the gas is shown in projection as a moment-1 map in Fig. 2a, with moment-0 contours in black for easy comparison. The moment-1 map was obtained after first blanking pixels in the spectral cube with an absolute value lower than $1.6 \mathrm{mJy} \mathrm{beam}^{-1}$. This corresponds to selecting emission and absorption stronger than $4 \sigma$, and was made to reduce influence from noise, which is also present at velocities where there is no real emission or absorption. Although the spatial and spectral resolution is limited, a range of velocities are clearly detected from about $2000 \mathrm{~km} \mathrm{~s}^{-1}$ to $2250 \mathrm{~km} \mathrm{~s}^{-1}$. This corresponds to both red- and blueshifted emission with respect to the velocity of $2088 \mathrm{~km} \mathrm{~s}^{-1}$ measured for NGC 4418 by Sakamoto et al. (2013).

\subsubsection{Position-velocity diagram and spectra}

Figure $2 \mathrm{~b}$ shows a position-velocity (PV) diagram extracted from the spectral cube along the dashed line shown in Fig. 2a. Four distinct features can be seen at the approximate relative offset positions of -200", -40" (VV 655), 152" (NGC 4418; in absorption), and $212^{\prime \prime}$. Spectra extracted at these positions, that is, the dashed lines in panel b, are presented in Fig. 3.

The four spectra were modelled using two Gaussian intensity distributions each. The best-fit parameters are listed in Table 1. Although limited by spectral and spatial resolution, we note a tentative double structure in the first two panels (offsets -200" and $\left.-40^{\prime \prime}\right)$. We interpret the average HI velocity in the second panel towards VV 655, of $2202 \pm 20 \mathrm{~km} \mathrm{~s}^{-1}$, as the systemic velocity of the galaxy where the uncertainty on the central velocity is chosen to cover the two tentative peaks in the spectrum. The third panel, towards NGC 4418, is best fitted with two absorption components. While the component of Costagliola et al. (2013) fitted at $2094 \pm 22 \mathrm{~km} \mathrm{~s}^{-1}$ is in excellent agreement with 


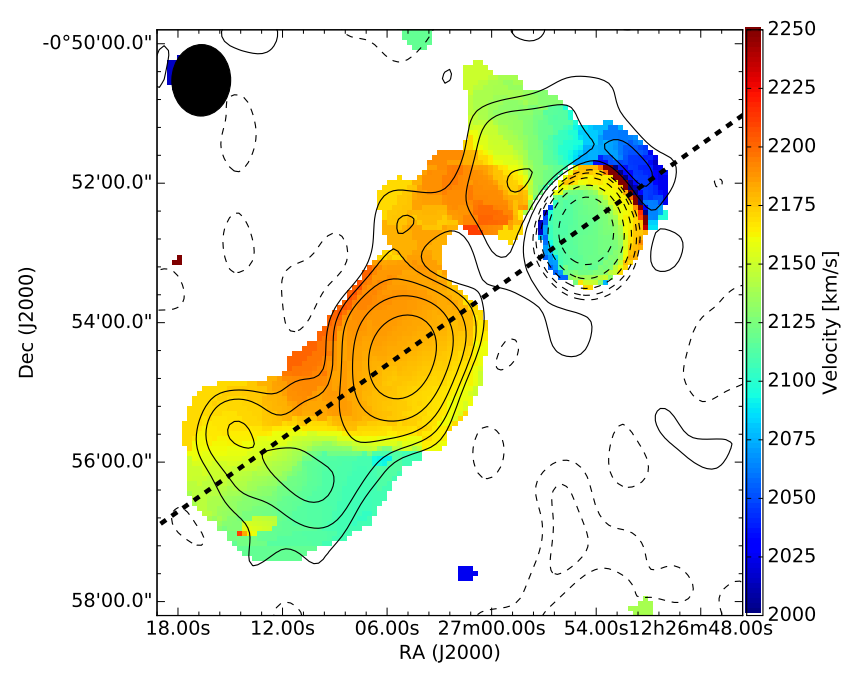

(a) HI moment-1 map with moment-0 contours

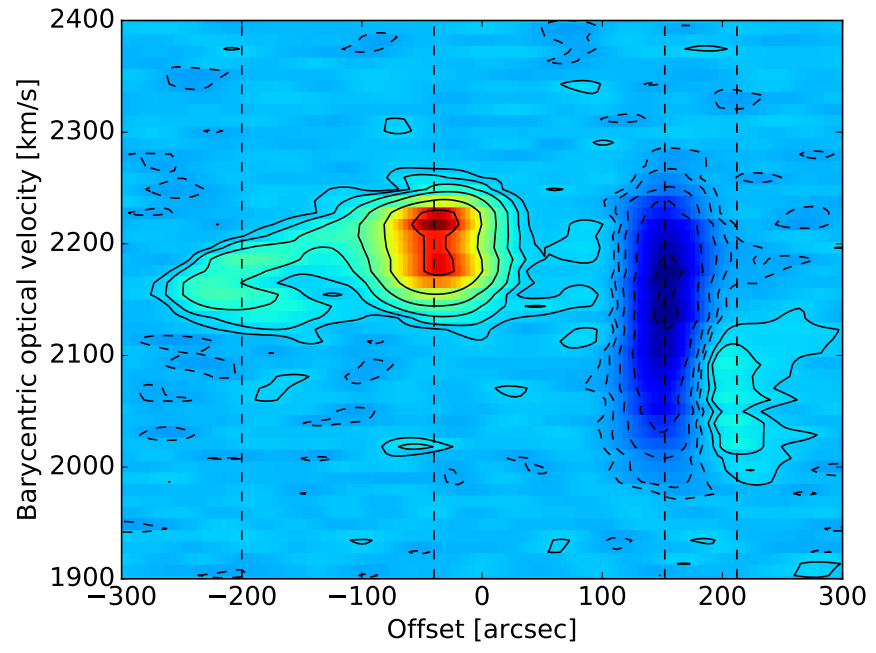

(b) PV-diagram along the dashed line in panel a.

Fig. 2. Panel $a$ : moment-1 HI map in colour with the moment- 0 contours from Fig. $1 \mathrm{~b}$ in black. The CLEAN beam is displayed as a black ellipse in the top left corner. Panel $b$ : position-velocity diagram extracted along the dashed line in panel a; an $11.3^{\prime}$ slice from RA $12^{\mathrm{h}} 27^{\mathrm{m}} 21^{\mathrm{s}} 547$ Dec $-00^{\circ} 57^{\prime} 19.85^{\prime \prime}$ to RA $12^{\mathrm{h}} 26^{\mathrm{m}} 44.380$ Dec $-00^{\circ} 50^{\prime} 48.73^{\prime \prime}$, that is, passing through both NGC 4418 and VV 655 . Four distinct features can be seen along the slice at the approximate relative offset values of $-200^{\prime \prime},-40^{\prime \prime}$ (VV 655), 152" (NGC 4418; in absorption), and 212". Spectra extracted at these positions, that is, the dashed lines in panel $b$, are presented in Fig. 3. Contours in panel $b$ are $\pm[0.025,0.05,0.1,0.2,0.4,0.8] \times$ $26.7 \mathrm{mJy} /$ beam (the peak).

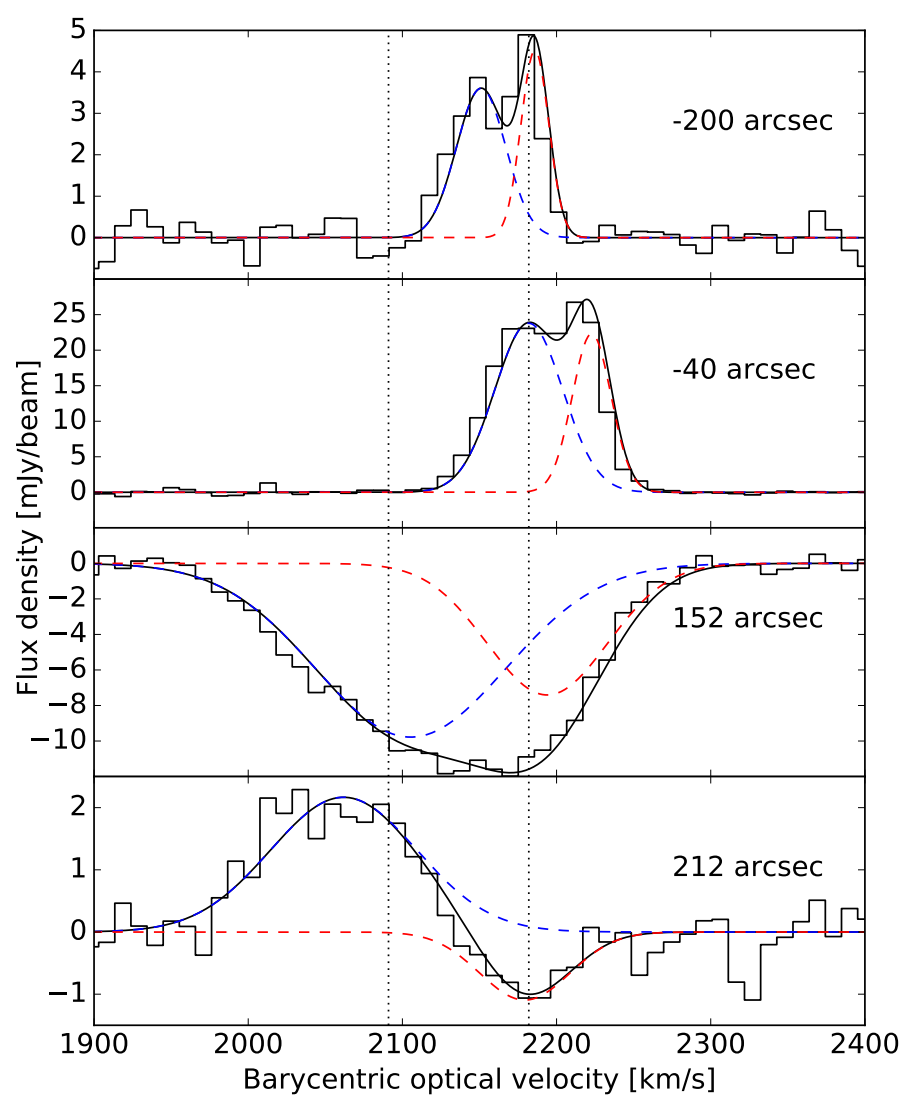

Fig. 3. Spectra extracted towards the four directions defined in Fig. $2 \mathrm{~b}$. The dashed curves show the fitted Gaussian components listed in Table 1. The solid line in each panel represents the sum of the fitted components, which are to be compared with the observed spectra in black steps. The vertical dotted lines show the velocities for the two components fitted to $\mathrm{CO}(2-1)$ by Costagliola et al. (2013). For reference, the systemic velocity of NGC 4418 has been measured as $2088 \mathrm{~km} \mathrm{~s}^{-1}$ by Sakamoto et al. (2013).
Table 1. Best-fit parameters for the spectra shown in Fig. 3.

\begin{tabular}{rrrrr}
\hline \hline $\begin{array}{r}\text { Offset } \\
{\left[{ }^{\prime}\right]}\end{array}$ & $\begin{array}{r}v_{\mathrm{c}} \\
{\left[\mathrm{km} \mathrm{s}^{-1}\right]}\end{array}$ & $\begin{array}{r}\text { Peak } \\
{[\mathrm{mJy} / \text { beam }]}\end{array}$ & $\begin{array}{r}F W H M \\
{\left[\mathrm{~km} \mathrm{~s}^{-1}\right]}\end{array}$ & $\tau_{\mathrm{p}}{ }^{a}$ \\
\hline-200 & $2151.1 \pm 2.3$ & $3.6 \pm 0.3$ & $37.7 \pm 5.4$ & - \\
-200 & $2186.0 \pm 1.2$ & $4.5 \pm 0.4$ & $21.3 \pm 2.5$ & - \\
\hline$-40^{b}$ & $2182.0 \pm 0.7$ & $23.8 \pm 0.2$ & $51.9 \pm 1.3$ & - \\
$-40^{b}$ & $2222.8 \pm 0.4$ & $22.3 \pm 0.6$ & $29.8 \pm 0.8$ & - \\
\hline $152^{c}$ & $2105.4 \pm 10.0$ & $-9.8 \pm 0.6$ & $145.1 \pm 12.8$ & 0.28 \\
$152^{c}$ & $2194.0 \pm 4.4$ & $-7.4 \pm 1.6$ & $92.2 \pm 8.3$ & 0.21 \\
\hline 212 & $2061.9 \pm 5.1$ & $2.2 \pm 0.2$ & $112.0 \pm 13.0$ & - \\
212 & $2179.4 \pm 7.7$ & $-1.1 \pm 0.2$ & $68.7 \pm 17.5$ & - \\
\hline
\end{tabular}

Notes. ${ }^{(a)}$ Assuming an unresolved background continuum of $40 \mathrm{mJy}$ from NGC 4418 as noted in Sect. 3.1. ${ }^{(b)}$ Towards VV 655. ${ }^{(c)}$ Towards NGC 4418

our result, their second $\mathrm{HI}$ component at $2153 \pm 11 \mathrm{~km} \mathrm{~s}^{-1}$ has a significantly lower velocity than we derived. This may be due to the fitting constraints employed by Costagliola et al. (2013), who did not fit the two components simultaneously. The fourth panel corresponds to a region north-west of NGC 4418. In addition to redshifted absorption, we also see prominent blueshifted emission.

Assuming the $\mathrm{HI}$ absorption from the $1.4 \mathrm{GHz}$ continuum of $40 \mathrm{mJy}$ dominates the HI emission towards NGC 4418, we can estimate the optical depth as $\tau \approx-\ln (1+p / 40 \mathrm{mJy})$, where $p$ are the (negative) peak values listed in Table 1 . The estimated $\tau$ for the two absorption components towards NGC 4418 are listed in Table 1. 


\subsection{2. $\mathrm{HI}$ emission bridge between NGC 4418 and VV 655}

In Sect. 3.3.1 we noted the systemic HI velocities measured for VV 655 and NGC 4418 of $2202 \pm 20 \mathrm{~km} \mathrm{~s}^{-1}$ and $2105.5 \pm$ $10 \mathrm{~km} \mathrm{~s}^{-1}$, respectively. The spectral cube reveals that HI emission connects VV 655 and NGC 4418. This structure is curved in three-dimensional (RA, Dec, Velocity) space and cannot be well illustrated by a moment map (i.e. Figs. $1 \mathrm{~b}$ and $2 \mathrm{a}$ ) or a single PV diagram (such as Fig. 2b). To show this three-dimensional structure in two dimensions, we plot three projections of the spectral cube in Fig. 4. In addition to the velocity axis, the velocity information is also reflected in the colour map. We note that the projections only show emission (not absorption), and only pixels brighter than $4 \sigma$, that is, $1.6 \mathrm{mJy} /$ beam, to reduce influence of noise. An animated GIF image showing more projections is available online. From the three-dimensional data it is clear that an HI emission bridge connects NGC 4418 and VV 655.

\subsection{Correction for $\mathrm{HI}$ absorption}

As noted in Sect. 3.1, the $1.4 \mathrm{GHz}$ continuum from NGC 4418 appears unresolved in these observations. However, the limited angular resolution of our data causes absorption towards the nucleus to blend outwards, reducing nearby emission. To obtain better estimates of the true HI emission, we therefore corrected for the unresolved absorption in the cube. This was done by assuming the absorption to be unresolved in each velocity channel, and therefore completely described by the value of the minimum pixel in each channel. To account for noise variations and pixel size limitations, we allowed the minimum position to vary between the channels. The variations were limited to the minor beam FWHM of 12 pixels around the NGC 4418 position, but the actual shift of the minimum position was less than 3 pixels between all channels.

In each velocity channel with negative values towards NGC 4418, we subtracted the CLEAN beam multiplied with the minimum pixel value, that is, assuming an unresolved background continuum. The strong absorption feature towards NGC 4418 was successfully removed (supporting our singlepixel assumption), and we now recover more $\mathrm{HI}$ emission close to NGC 4418, see Fig. 5 and compare with Fig. 1b. While we restricted our subtraction to pixels with negative values, there may still be absorption present that reduces the emission in some channels, although not below zero, that is to say, the emission is not optically thin. Furthermore, we note that our method of correcting for the absorption cannot recover any $\mathrm{HI}$ emission from the nucleus of NGC4418. These effects may explain the remaining weak depression in the emission towards NGC 4418 in Fig. 5.

\section{5. $\mathrm{HI}$ emission fluxes and mass estimates}

To measure the integrated flux density of the HI emission, we have divided the emission into three regions in Fig. 5: north, defined as the top-right polygon, includes emission associated with NGC 4418. The middle polygon includes emission associated with VV 655, and the south polygon includes emission associated with the south-east extension from VV 655. The correction for absorption towards NGC 4418 makes it possible to estimate the HI gas mass from emission associated also with NGC 4418. This value is a lower limit since the emission may not be optically thin, as noted in Sect. 3.4. The HI emission flux for the three regions was measured by summing pixels in a moment- 0 map, and they are listed in Table 2 . We note that
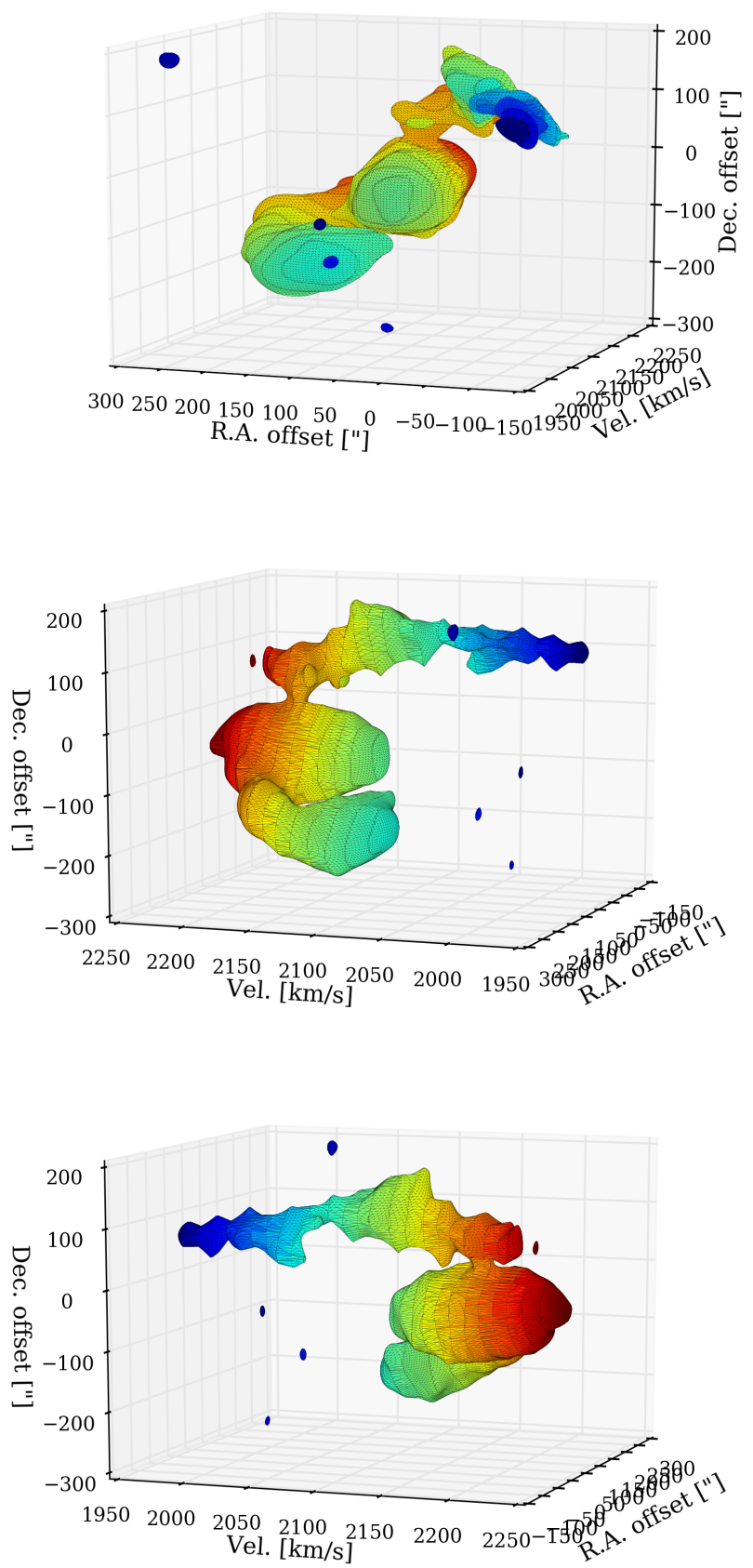

Fig. 4. Three projections of the continuum-subtracted HI data cube (RA, Dec, velocity) showing the arc-like connection between NGC 4418 and VV 655 in HI emission. For reference, the systemic HI velocities for VV 655 and NGC 4418 are $2202 \pm 20 \mathrm{~km} \mathrm{~s}^{-1}$ and $2105.5 \pm 10 \mathrm{~km} \mathrm{~s}^{-1}$, respectively (see Sect. 3.3.1). Note that this figure only shows the emission, not the absorption towards NGC 4418.

our value for VV 655 is higher than the $2.0 \pm 0.7 \mathrm{Jy} \mathrm{km} \mathrm{s}^{-1}$ reported by Thomas et al. (2002) using 12 min of C-array VLAdata. However, this discrepancy can be explained given the limited sensitivity in such a snapshot, in combination with the fact that VV 655 may be partly resolved out by the slightly longer baselines.

From the detected HI emission we estimate the HI mass using Eq. (13.57) in Wilson et al. (2009):

$M_{\mathrm{HI}}\left[M_{\odot}\right]=2.36 \times 10^{5} D^{2} \int S_{\nu} \mathrm{d} v$, 


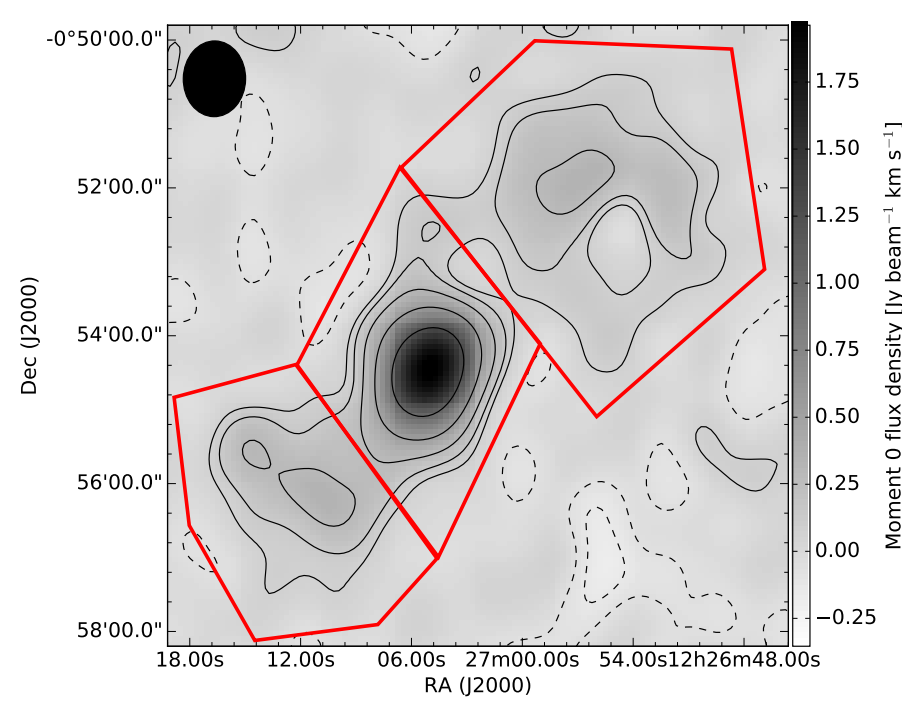

Fig. 5. Moment -0 map of $\mathrm{HI}$ after correcting for the absorption towards NGC 4418, as described in Sect. 3.4. More emission is seen close to NGC 4418 than in Fig. 1b. The red polygons mark three regions where pixels were summed to measure the flux values listed in Table 2. Both greyscale and contours show the moment- 0 map, with the same contours as in Fig. 1b.

Table 2. Integrated HI emission flux measurements for the three regions defined in Fig. 5 together with corresponding HI mass estimates from Eq. (1).

\begin{tabular}{l|r|r}
\hline \hline Region & Flux [Jy km s $\left.{ }^{-1}\right]$ & $M_{\mathrm{HI}}\left[M_{\odot}\right]$ \\
\hline North (NGC 4418) & 1.47 & $4.0 \times 10^{8}$ \\
Middle (VV 655) & 4.05 & $11.0 \times 10^{8}$ \\
South & 0.83 & $2.3 \times 10^{8}$ \\
\hline Sum & 6.35 & $17.3 \times 10^{8}$ \\
\hline
\end{tabular}

Notes. Note that these values were obtained after removing the absorption towards NGC 4418, as described in Sect. 3.4.

where $\int S_{\nu} \mathrm{d} v$ is integrated line flux in $\mathrm{Jy} \mathrm{km} \mathrm{s}^{-1}$, and $D$ is the distance in Mpc. With $D=34 \mathrm{Mpc}$, we obtain the values listed in Table 2. We note that the estimated HI mass of VV 655 is very similar to the HI masses of other blue compact dwarf galaxies measured by Thuan et al. (2016).

\section{Discussion}

In this section we discuss the results presented in the previous section.

\subsection{NGC 4418 and VV 655 are interacting}

The spectral cube presented in Sect. 3.3.2 shows that NGC 4418 and VV 655 are connected by a bridge of atomic hydrogen seen in emission. Furthermore, the similar velocities of the gas surrounding VV 655 and NGC 4418 and the elongated gas can be interpreted as signatures of a recent or ongoing interaction process, where the elongated $\mathrm{HI}$ structure connecting the two galaxies may have formed when the more massive NGC 4418 disturbed the HI surrounding VV 655. We propose that at some time, relatively recently, NGC 4418 was gas poor, while VV 655 had a significant HI envelope. As the galaxies passed each other, the more massive NGC 4418 tidally stripped away HI gas from
VV 655. This interaction formed the elongated HI structure we see today. The HI morphology is indeed similar to the structure seen in other interacting pairs in the HI Rogues Gallery (Hibbard et al. 2001). For example, the interacting galaxy pair II ZW 70/71 (UGC 9562 and UGC 9560) have an HI emission bridge connecting the two galaxies and an $\mathrm{HI}$ extension from one of the galaxies (Cox et al. 2001).

The interaction morphology significantly depends on the mass ratio of the two interacting galaxies and the relative alignment of the galaxies spin with the orbital angular momentum. The (baryonic) mass ratio of the system can be derived from the HI and some optical and near-infrared (NIR) photometry. The stellar mass ratio of NGC 4418 vs. VV 655 is $\sim 85: 1$ (SDSS DR12, Maraston et al. 2009), but including the HI from Table 2 brings the mass ratio to $\sim 8: 1$. This should result in a merger similar to Toomre \& Toomre (1972). We interpret the observed HI bridge between VV 655 and NGC 4418, and the HI tail southeast of VV 655, as tidal features caused by the interaction.

Since there is one prominent tail and the galaxies are well separated, it is very likely that we are viewing the system after first pass, but before a second close pass. The existence of a welldeveloped tail argues in favour of the galaxies having passed at least once (Toomre \& Toomre 1972). However, the first pass typically removes enough angular momentum from the orbit that the second encounters are more radial, and then dynamical friction prevents them from reaching large separations after second pass. The well-defined tail also suggests that the discs did not have a deeply interpenetrating encounter, as that tends to significantly disturb or destroy discs rather than draw out long tails.

The prominence of the tidal tail and the well-defined bridge mean that VV 655 very likely had a prograde encounter, where the angular momentum of the VV 655 disc was aligned (within $90^{\circ}$ ) with the orbital angular momentum of the pair. This offers some constraints on the geometry of the system. However, because NGC 4418 does not have (as prominent) a dynamically cold disc, we would not necessarily expect it to show a tail and bridge (in stars) if it was a prograde encounter. Given the lack of clear tidal features from NGC 4418, the data are also consistent with a retrograde (e.g., misaligned, $i>90^{\circ}$ ) encounter. It is therefore challenging, given the available data, to constrain the inclination of the NGC 4418 disc relative to the orbital plane of the interaction (e.g. Fig. 6a by Toomre \& Toomre 1972).

\subsection{Time since first pass}

We can estimate the dynamical age of the tail and by proxy the time since the first close pass of the two galaxies. Using the length of the tidal tail and the velocity difference between the tail and the main body of VV 655, we estimate the age as

$t=0.8 \cdot r_{\mathrm{p}} v^{-1} \cot (\beta)$,

where $r_{\mathrm{p}}$ is the projected distance between the centre of VV 655 to the tip of the tail (TT), $v$ is the relative velocity between VV 655 and TT, and $\beta$ is the angle between our line of sight and the vector extending from VV 655 to TT. Schweizer (1982) first used this for NGC 7252, and age estimates of this type are generally found to be consistent with the ages estimated from dynamical modelling (e.g. Hibbard \& Mihos 1995, for NGC 7252). From our data we measure TT at RA $12^{\mathrm{h}} 27^{\mathrm{m}} 10^{\mathrm{s}}$, Dec $-00^{\circ} 56^{\prime} 20^{\prime \prime}$ with a velocity of $2097 \mathrm{~km} \mathrm{~s}^{-1}$. The centre of VV 655 is at RA $12^{\mathrm{h}} 27^{\mathrm{m}} 05^{\mathrm{s}}$, Dec $-00^{\circ} 54^{\prime} 20^{\prime \prime}$ with a systemic velocity of $2202 \mathrm{~km} \mathrm{~s}^{-1}$ (Sect. 3.3.1). This implies $r_{\mathrm{p}} \approx$ $2.35^{\prime} \approx 22.8 \mathrm{kpc}$ and $v \approx 105 \mathrm{~km} \mathrm{~s}^{-1}$. The angle $\beta$ is difficult 
to obtain, but from Fig. 4 we estimate $\beta \approx 45^{\circ}$ within a factor of 2. Equation (2) then gives an estimate of the dynamical age of the tail of $\sim 190$ Myr. Following Schweizer (1982), we note that this estimate is uncertain by a factor of about 2 because of the uncertain projection angle. We note that the age is similar to the first-pass ages obtained through detailed modelling of other well-known merger pairs by Privon et al. (2013). Detailed dynamical modelling of the interaction between NGC 4418 and VV 655, preferably also using data with higher spatial and spectral resolution, may give more insight regarding the interaction scenario, but this is beyond the scope of this paper.

\subsection{HI falling towards NGC 4418}

The redshifted absorption detected towards NGC 4418 (see panel 3 in Fig. 3) implies gas in front of NGC 4418 travelling towards the galaxy. The velocity of about $90 \mathrm{~km} \mathrm{~s}^{-1}$ relative to NGC 4418 is indeed similar to the redshifted $\mathrm{CO}(2-1)$ component found by Costagliola et al. (2013). This is consistent with infall, but because of the limited spatial resolution, we cannot probe the detailed dynamics around NGC 4418. While a significant amount of $\mathrm{HI}$ gas is falling towards NGC 4418, it is not clear from these data whether this gas does fall all the way into the centre of the galaxy. However, if some of the gas does reach the centre, it can explain the recent star formation and/or AGN activity in the galaxy nucleus.

\subsection{North-west outflow}

North-west of NGC 4418, we detect HI in emission blueshifted with respect to the systemic velocity of the galaxy. The blueshift can be explained if the gas is being pushed with an inclination towards us, that is, out from the galaxy nucleus. The orientation of this emission is consistent with being a large-scale continuation of the outflow signature seen by Sakamoto et al. (2013), their Fig. 16. It is possible that the activity in the nucleus is powering an outflow that expels HI gas surrounding the galaxy. This argument is supported by the fact that there is no other source visible in this region that could be the source of this emission. Such an outflow can be driven by either intense star formation or AGN activitiy, or a combination of both.

To measure the HI emission associated with the outflow, we used the absorption-subtracted image cube (see Sect. 3.4) to produce a moment-0 map, including only the channels containing blueshifted emission associated with the outflow position. This moment-0 map is shown in greyscale in Fig. 6. Summing the pixels in the marked region in Fig. 6, we measure a flux of $0.48 \mathrm{Jy}_{\text {beam }}^{-1} \mathrm{~km} \mathrm{~s}^{-1}$ associated with the outflow, which using Eq. (1) translates into an atomic hydrogen gas mass of $1.3 \times 10^{8} M_{\odot}$.

We note that the outflow is not clearly resolved in most velocity channels. As an approximation of the outflow volume, we assume a cylinder with diameter $25^{\prime \prime}$ (equal to half the minor axis of the CLEAN restoring PSF) and length 60" (equal to the distance from the centre of NGC 4418 to the peak of the outflow emission in Fig. 6). Given the HI mass estimate above, this implies an average outflow density of $1.1 \times 10^{6} M_{\odot} \mathrm{kpc}^{-3}$. This is an upper limit, given that the outflow structure is likely conical instead of a cylinder, as evident from Sakamoto et al. 2013, their Fig. 16.

To estimate the mass outflow rate. we need the deprojected outflow velocity. As an upper limit, we take the velocity of

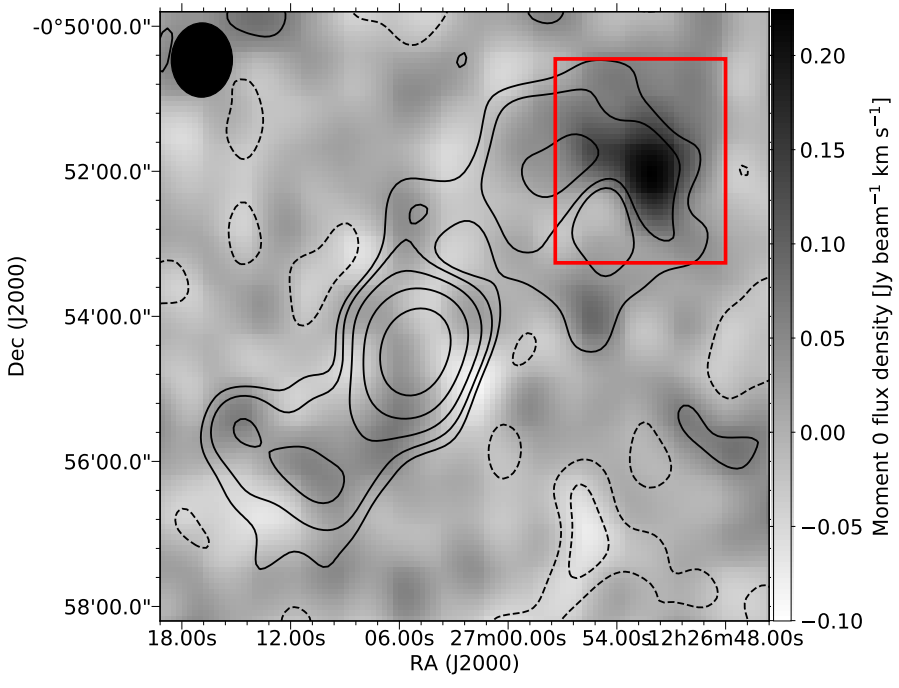

Fig. 6. In greyscale: moment-0 map of 19 most blueshifted channels, after first removing the absorption towards NGC 4418, as described in Sect. 3.4. Contours are the same as in Fig. 5 to guide the eye. The red polygon marks regions where pixels were summed to measure the flux of the outflow, discussed in Sect. 4.4.

the most blueshifted velocity channel with emission brighter than $3 \sigma$, which gives a velocity of $2004 \mathrm{~km} \mathrm{~s}^{-1}$. (This velocity can also be approximately determined from Fig. $2 b$ or the lowest panel in Fig. 3, but using the full cube provides maximum precision.) Taking the systemic velocity for NGC 4418 of $2088 \mathrm{~km} \mathrm{~s}^{-1}$ from Sakamoto et al. (2013) implies an outflow speed of $84 \mathrm{~km} \mathrm{~s}^{-1}$. Assuming that the outflow is perpendicular to the galaxy disc, which has an inclination angle of $62^{\circ}$ (Sakamoto et al. 2013), we obtain a deprojected outflow velocity of $178 \mathrm{~km} \mathrm{~s}^{-1}$. We note that this is similar to the projected minoraxis velocity shear of $200 \mathrm{~km} \mathrm{~s}^{-1}$ measured in $\left[\mathrm{N}_{\mathrm{II}}\right]$ as noted by Sakamoto et al. (2013).

Given the above assumptions, we estimate a mass outflow rate of $2.5 M_{\odot} \mathrm{yr}^{-1}$. This estimate is to be taken as an order-ofmagnitude estimate. More accurate constraints require observations with higher spatial resolution and sensitivity to properly study the HI velocity structure on scales between those sampled by MERLIN, as presented by Costagliola et al. (2013), and those sampled by VLA, as presented in this work.

\section{Summary}

We presented observations at $1.4 \mathrm{GHz}$ with the VLA of radio continuum, as well as emission and absorption from atomic hydrogen, towards the galaxies NGC 4418 and VV 655. We detected a large $\left(5^{\prime} \sim 50 \mathrm{kpc}\right) \mathrm{HI}$ structure in emission, connecting the two galaxies and extending south-east from VV 655. While NGC 4418 is bright in continuum emission and seen in HI absorption, VV 655 is barely detected in the continuum, but shows bright $\mathrm{HI}$ emission $\left(M_{\mathrm{HI}} \sim 10^{9} M_{\odot}\right)$. We estimated SFRs from the $1.4 \mathrm{GHz}$ continuum of $3.2 M_{\odot} \mathrm{yr}^{-1}$ and $0.13 M_{\odot} \mathrm{yr}^{-1}$ for NGC 4418 and VV 655, respectively.

We fitted Gaussian intensity distributions to spectra extracted from the image cube and measure systemic HI velocities of $2202 \pm 20 \mathrm{~km} \mathrm{~s}^{-1}$ (emission) and $2105.4 \pm 10 \mathrm{~km} \mathrm{~s}^{-1}$ (absorption) for VV 655 and NGC 4418, respectively. We also detected redshifted $\mathrm{HI}$ absorption $\left(v_{\mathrm{c}}=2194.0 \pm 4.4 \mathrm{~km} \mathrm{~s}^{-1}\right)$ towards NGC 4418, which suggests gas infall. The morphology and velocity structure seen in $\mathrm{HI}$ is consistent with an interaction 
scenario, where gas was transferred from VV 655 to NGC 4418. Some gas is falling towards NGC 4418 and may fuel the activity in the centre. From the extent and relative velocity of the VV 655 HI tail, we estimate that the two galaxies first passed $\sim 190 \mathrm{Myr}$ ago.

North-west of NGC 4418, we detected HI in emission, blueshifted with respect to NGC 4418, which is consistent with an outflow perpendicular to the galaxy disk. We interpreted the blueshifted HI-emission north-west of NGC 4418 as a continuation of the outflow previously discussed by Sakamoto et al. (2013), powered by star formation and/or black hole accretion in the centre. The observed maximum velocity of $v_{\mathrm{c}}=2004 \mathrm{~km} \mathrm{~s}^{-1}$ implies a deprojected speed of $187 \mathrm{~km} \mathrm{~s}^{-1}$. Assuming a steadystate cylindrical outflow from the core of NGC 4418, we obtain an order of magnitude estimate of the HI mass outflow rate as $2.5 M_{\odot} \mathrm{yr}^{-1}$.

Acknowledgements. F.C. acknowledges support from Swedish National Research Council grant 637-2013-7261. G.C.P. was supported by a FONDECYT Postdoctoral Fellowship (No. 3150361). This research made use of APLpy, an open-source plotting package for Python (Robitaille \& Bressert 2012). Using data from the Karl G. Jansky Very Large Array (VLA), we acknowledge that the National Radio Astronomy Observatory is a facility of the National Science Foundation operated under cooperative agreement by Associated Universities, Inc. Funding for SDSS-III has been provided by the Alfred P. Sloan Foundation, the Participating Institutions, the National Science Foundation, and the U.S. Department of Energy Office of Science. The SDSS-III web site is http://www.sdss3.org/. The authors acknowledge assistance from Joachim Wiegert and Niklas Falstad with obtaining Herschel $70 \mu \mathrm{m}$ SFR estimates. The authors also wish to thank John Hibbard for discussions during the preparation of this manuscript. Finally, the authors acknowledge the constructive feedback offered by the anonymous referee that helped to improve the quality of this paper.

\section{References}

Alam, S., Albareti, F. D., Allende Prieto, C., et al. 2015, ApJS, 219, 12 Baan, W. A., \& Klöckner, H.-R. 2006, A\&A, 449, 559
Becker, R. H., White, R. L., \& Helfand, D. J. 1995, ApJ, 450, 559

Bell, E. F. 2003, ApJ, 586, 794

Calzetti, D., Wu, S.-Y., Hong, S., et al. 2010, ApJ, 714, 1256

Colless, M., Peterson, B. A., Jackson, C., et al. 2003, ArXiv e-prints [arXiv:astro-ph/0306581]

Costagliola, F., Aalto, S., Sakamoto, K., et al. 2013, A\&A, 556, A66

Costagliola, F., Sakamoto, K., Muller, S., et al. 2015, A\&A, 582, A91

Cox, A. L., Sparke, L. S., Watson, A. M., \& van Moorsel, G. 2001, AJ, 121, 692

Evans, A. S., Becklin, E. E., Scoville, N. Z., et al. 2003, AJ, 125, 2341

González-Alfonso, E., Fischer, J., Graciá-Carpio, J., et al. 2012, A\&A, 541, A4

Greisen, E. W. 2003, Information Handling in Astronomy - Historical Vistas, 285,109

Hibbard, J. E., \& Mihos, J. C. 1995, AJ, 110, 140

Hibbard, J. E., van Gorkom, J. H., Rupen, M. P., \& Schiminovich, D. 2001, in Gas and Galaxy Evolution, eds. J. E. Hibbard, M. Rupen, \& J. H. van Gorkom, ASP Conf. Ser., 240

Kawara, K., Taniguchi, Y., Nakai, N., \& Sofue, Y. 1990, ApJ, 365, L1

Kettenis, M., van Langevelde, H. J., Reynolds, C., \& Cotton, B. 2006, in Astronomical Data Analysis Software and Systems XV, eds. C. Gabriel, C. Arviset, D. Ponz, \& S. Enrique, ASP Conf. Ser., 351, 497

Lehnert, M. D., \& Heckman, T. M. 1995, ApJS, 97, 89

Maraston, C., Strömbäck, G., Thomas, D., Wake, D. A., \& Nichol, R. C. 2009, MNRAS, 394, L107

Pilbratt, G. L., Riedinger, J. R., Passvogel, T., et al. 2010, A\&A, 518, L1

Poglitsch, A., Waelkens, C., Geis, N., et al. 2010, A\&A, 518, L2

Privon, G. C., Barnes, J. E., Evans, A. S., et al. 2013, ApJ, 771, 120

Robitaille, T., \& Bressert, E. 2012, APLpy: Astronomical Plotting Library in Python, Astrophysics Source Code Library [record ascl : 1208.017]

Roche, P. F., Aitken, D. K., Smith, C. H., \& James, S. D. 1986, MNRAS, 218, 19P

Sakamoto, K., Aalto, S., Costagliola, F., et al. 2013, ApJ, 764, 42

Schweizer, F. 1982, ApJ, 252, 455

Spoon, H. W. W., Keane, J. V., Tielens, A. G. G. M., Lutz, D., \& Moorwood, A. F. M. 2001, A\&A, 365, L353

Thomas, H. C., Dunne, L., Clemens, M. S., et al. 2002, MNRAS, 329, 747

Thuan, T. X., Goehring, K. M., Hibbard, J. E., Izotov, Y. I., \& Hunt, L. K. 2016 , MNRAS, 463, 4268

Toomre, A., \& Toomre, J. 1972, ApJ, 178, 623

Varenius, E., Conway, J. E., Martí-Vidal, I., et al. 2014, A\&A, 566, A15

Wilson, T. L., Rohlfs, K., \& Hüttemeister, S. 2009, Tools of Radio Astronomy (Berlin: Springer-Verlag)

Yun, M. S., Reddy, N. A., \& Condon, J. J. 2001, ApJ, 554, 803 